\title{
Using Cisco Packet Tracer to simulate Smart Home
}

\author{
Ghaliya Alfarsi \\ Information Technology department \\ AlBuraimi College \\ Buraimi, Oman \\ Ragad M Tawafak \\ Information Technology department \\ AlBuraimi College \\ Buraimi, Oman \\ Abir Alsidiri \\ Information Technology department \\ AlBuraimi College \\ Buraimi, Oman
}

\author{
Jasiya Jabbar \\ Information Technology department \\ AlBuraimi College \\ Buraimi, Oman \\ Sohail Iqbal Malik \\ Information Technology department \\ AlBuraimi College \\ Buraimi, Oman \\ Maryam Alsinani \\ Information Technology department \\ AlBuraimi College \\ Buraimi, Oman
}

\begin{abstract}
Technology plays a critical role in all daily activities of the present day. One of these needs is to create a smart home that controls operation and turns off electronic devices via a smartphone. This implementation can be implemented effectively using package tracking software that includes IoT functions to control and simulate a smart home. IoT technology can be applied to many real life issues, such as: homework, treatment, campus, office, etc. In this paper, the focus is on a safe home system that includes devices such as: air conditioning, alarm, lighting, and doors. Garage that is some of the day to day issues. The aim of this research is to come up with a simulation of smart devices that can be controlled by the end-user smart device remotely and then show the concept called smart home. Use of Cisco Packet Tracking Features Simulated smart home and IoT devices are monitored. Simulation results show that smart objects can be connected to the home portal and objects can be successfully monitored which leads to the idea of real life implementation.
\end{abstract}

Keywords- IOT technology, Cisco Packet Tracer, Home gateway, IoT server, IoT moniotor

\section{INTRODUCTION}

IOT means "Internet of Things" that defines things and their connections to the Internet (A \& K. A) (R. Pivare, M. Tazil 2011). The Internet of Things enables control of local and remote objects by consuming the integration of network technology. IoT connects devices around us as well as over the Internet and automates communications (Moinar, Shamsi, and Navar, 2012).

Groups of scientists and organizations have attempted to clarify the Internet of Things and define their proposed definition as [Halleret al] "a world where material things are seamlessly integrated into the information network and where material things can become an active participant in the business process." With the emergence of life changing on the Internet, the Internet of Things is expected to have a huge impact on life (Iova,2014).

Cisco packet tracer is a cisco multi-platform simulation tool to allow learners to simulate networks. It also helps generating IoT reformations. In our research work we used the latest version of cisco packet tracer. Fig 1 shows its interface.

Some of the objectives of cisco packet tracer are:

- Allows students to design complex networks

- Can be used a learning aid among students

- Allows students to explore IoT concepts

- Allows users to build, design and configure smart city and smart home by providing a different intelligent object that uses them.

- Provides realistic visualization and simulation of IoT devices

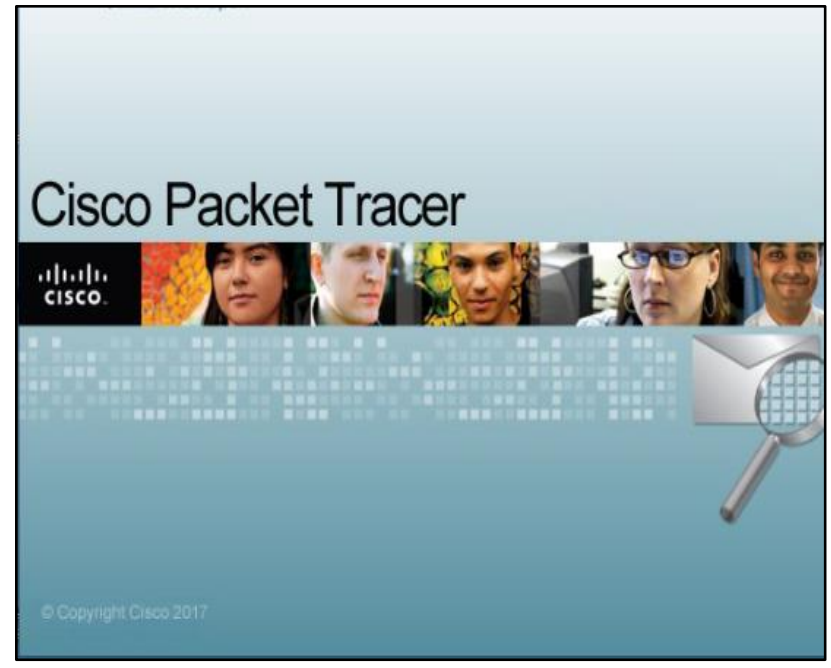

Fig. 1: Cisco Packet Tracer interface 
This research paper is organized into a number of sections which include literature review of related works, followed by methodology used in this development. Further the safe home implementation procedures and results are discussed. Finally the conclusion and references are included.

\section{LITERATURE REVIEW}

The smart home consist of the opportunity modernization of the smart learning to improve the smart devices about this part some Literature review were provided

Alexandria Al-oisi at 2014 studied the relationship between the Internet of Things and wireless sensor networks from the piles of ACOAP transport network objects. This standard provides compatibility between the network of things and the remote sensing system. On the way to do this, the first step of motivation organized the knowledge of IOT, further how sensors can be linked to collect information taking into account the ultimate goal of smart campus. The work utilized the smart COAP (communication constrained protocol) protocol in implementation (Alexandria, 2014).

Ahmed Abdi in 2018 conducted research on IoT and introduced the concept of smart university. It utilizes technology in designing a campus where some of the campus components to be replaced with the smart devices so as to simplify the work done. Cisco packet tracer was used to design a smart office where staff can control the office using their smart phones. IoT gateways provide the link between the smart office and internet through cloud. The objective of this study was to enrich University in a smart mode and use globalization and to connect all devices to the network and enable sensors to help and control the devices. It also allows the collaboration of sensors with devices in the best manner to provide high quality and technology based infrastructure in the university (Abdi, 2018) (Marian, 2015).

Miluzzo Choudhury and A. T. Campbell in 2010 conducted a questionnaire-based survey on mobile phone sensor. The smartphone has many built-in sensors, which is why it is called the most important sensor today. It has a motion sensor, GPS sensor, light sensor, and magnetism. These things are mainly used in different areas of IOT applications. There are different sensors used to measure temperature, pressure, humidity, medical, and so on smartphones are mainly used to control access and control of smart devices (Nicholas D. Lane, Emiliano Miluzzo, Hong Lu, Daniel Peebles, Tanzeem Choudhury, \& Andrew T. Campbell, 2010) (ALFarsi, Jabbar, \& ALSinani, 2018) (AlFarsi \& ALSinani, 2017).

Isa Al-Shemsi at in 2018 used the revised version of packet tracer in implementing smart home. This version also provided programming environment for controlling objects. To enable wireless sensor and smart objects, Home Gateway and microcontroller (MCU) were employed. Devices were registered using home gateway and MCU was used to link sensors to device. Even though programming language support was provided by the cisco packet tracer, the researcher used Java Script and Python for implementation. (Al-Shemsi, 2018).
Yin Jie et al in 2013 introduced the idea of smart home with IOT. They introduced architecture for the system, in which many applications can be integrated through its interface. RFID tags are exploited to ease the agent communication with the devices. The main issues to be resolved related to smart home is also included in this research (Jie, Pie, Jun, Yun, \& Wei, 2013).

Jetendra Joshi et al proposed an IOT based smart home which can control, monitor and access multiple devices from anywhere and anytime. They utilized Raspberry Pi and web server. To monitor home appliances, Raspberry Pi and Arduino was incorporated with Nrf modules. The readings are send to web server and it is monitored to give alarms under the influence of threats (Jetendra , et al., 2017)

\section{METHODOLOGY}

The idea of smart is very catchy and review on similar researches was conducted to understand its concepts and its architecture. As implemented by majority of researchers, cisco packet tracer was used for implementing smart home. The newly released cisco version was used as it supports home security features as well as provides programming environment besides networking features. The different programming languages supported are visual basic, java scripts, and python. Smartphone and home gateway are used to control the devices such as smart window, smart fan, smart garbage and sensor. The smart devices are connected to the IOT home gateway ports and smart phone is used to communicate with the smart devices.

\section{SAFE HOME DEVELOPMENT}

Home gateway has four Ethernet ports and wireless access point configured with the "Home Gateway" service set identifier (SSID). All the devices are connected to gateway using config menu in each device, the name of the gateway has to entered. This can be repeated to all sets of devices that has to be controlled in a home gateway. Ip config menu displays the lidt of devices connected to the home gateway. The smart phone is used to control all the devices.

The cisco packet tracer interface provides inbuilt devices to be added in the network. The first step is to select home gateway device from the network devices. To authenticate and validate the wireless connection, we also can configure home gateway with WEP/WPA-PSK /WPA2 protocols.

The second step in simulating a smart home is to connect devices to the home gateway. In this work, six wireless devices were coupled with the home gateway. The devices configured included fan, webcam, door, air condition, garage and motion detector. Figure 2 shows the smart objects connected in our system which included only wireless devices. 


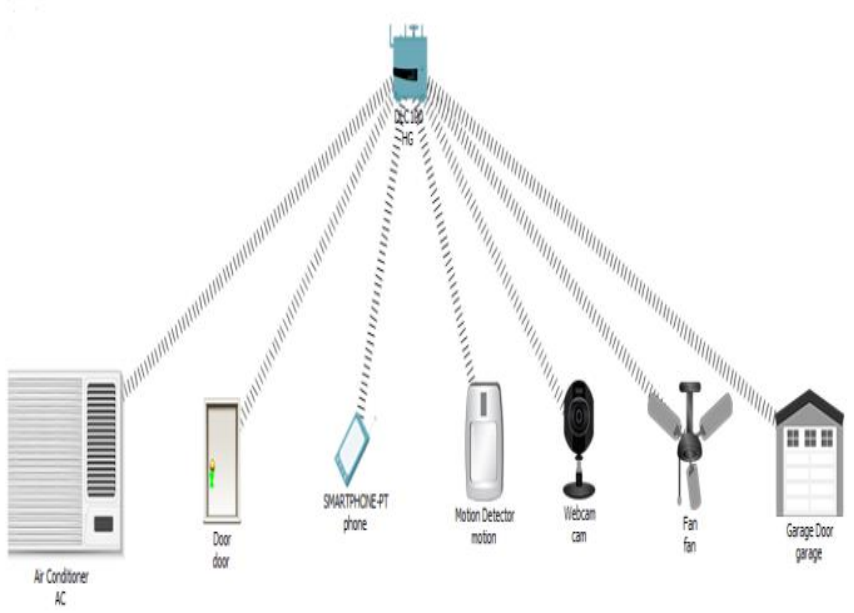

Fig. 2: Home gateway with six smart objects and smart mobile

In order to connect an object, the home object icon has to be selected from the device-type selection box. For example let us consider connecting $\mathrm{AC}$ to the home gateway. Select AC icon to the workspace and click it to open various settings of the device. In config tab, name of the device can be updated with the name you need to be displayed. Wireless0 has to be selected to enter SSID of home gateway. This to indicate that the device connected to the home

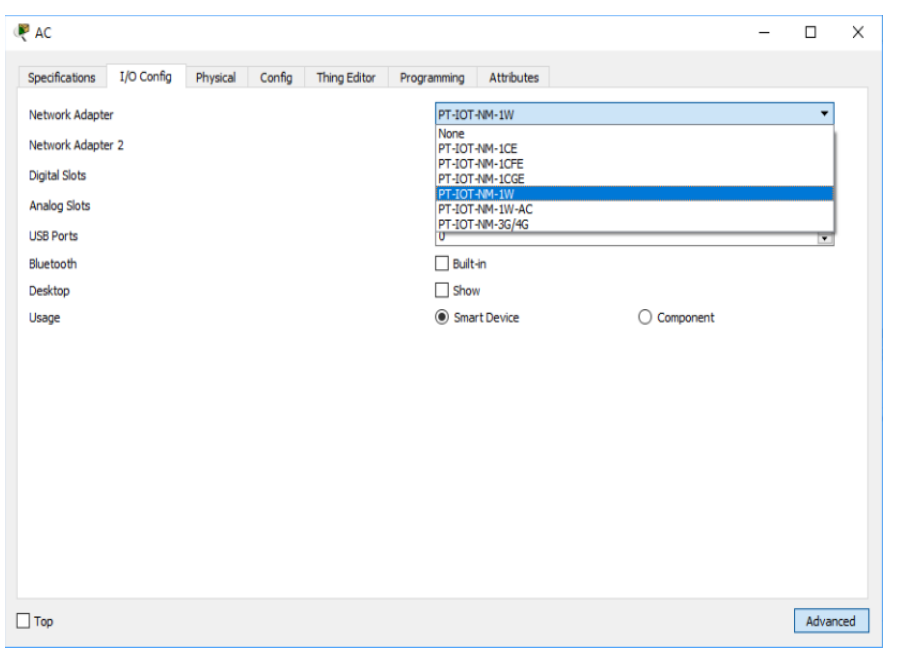

Fig. 3:AC setting.

gateway and connected to network wireless. The default gateway is 192.168.25.1. Fig. 3 shows the settings when AC is clicked which has advanced options.

There is another option called advanced at the bottom which gives additional menus. Here in I/O config tab, the network adapter setting has to be set to PT-IOT-NW-1W. Fig 4 shows the I/O config settings of advanced option of each smart object.

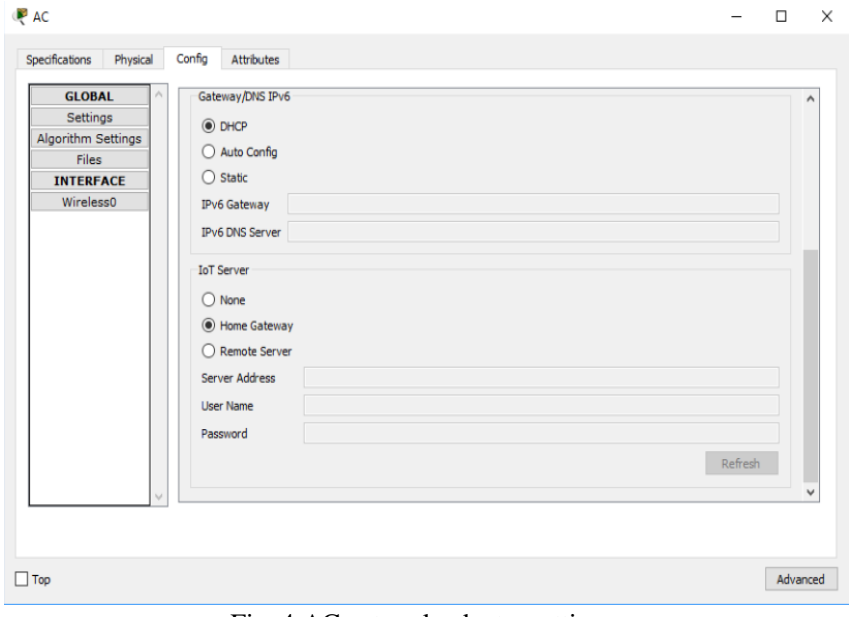

Fig. 4:AC network adapter setting.

These steps are repeated to add all the objects such as door, fan, motion detector, webcam and garage door. The user will control all devices by the smartphone. Fig.5 shows the features and settings specification of AC such as the name of the port, link, IP address, IPV6 address and the IP of the gateway after connecting to home gateway.

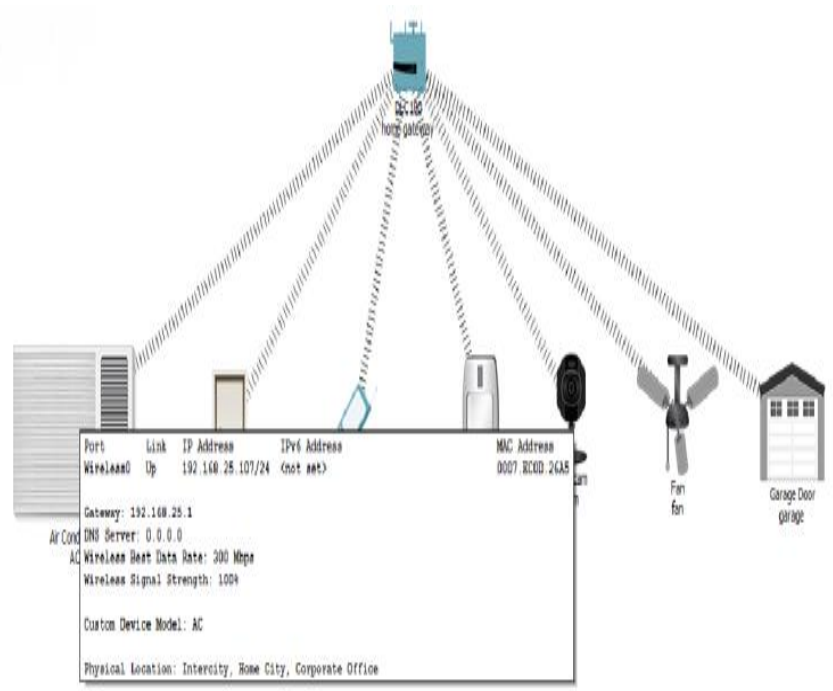

Fig. 5: AC specification

The next step is to connect the smart phone to the home gateway. Select the device type from the end devices selection box. When the smartphone icon is clicked, the configuration window appears. The SSID from Wireless0 has to be updated with home gateway instead of default and it leads to automated IP address generation for the smartphone. 


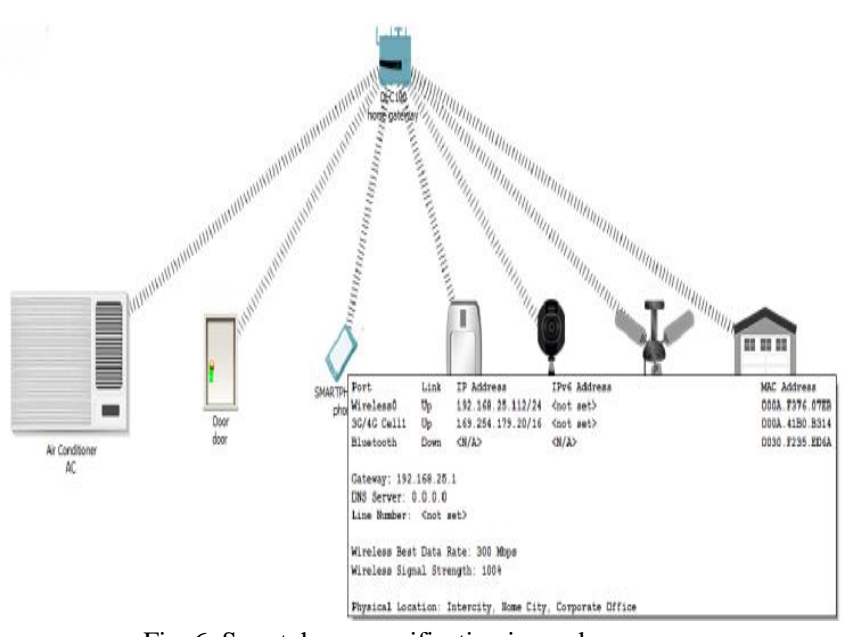

Fig. 6: Smartphone specification in workspace

The next step is to add rules and conditions for the smart objects to give notifications or alarm. In order to do this, from smartphone select the desktop tab and click IoT monitor. Fig. 7 shows the window that appears.

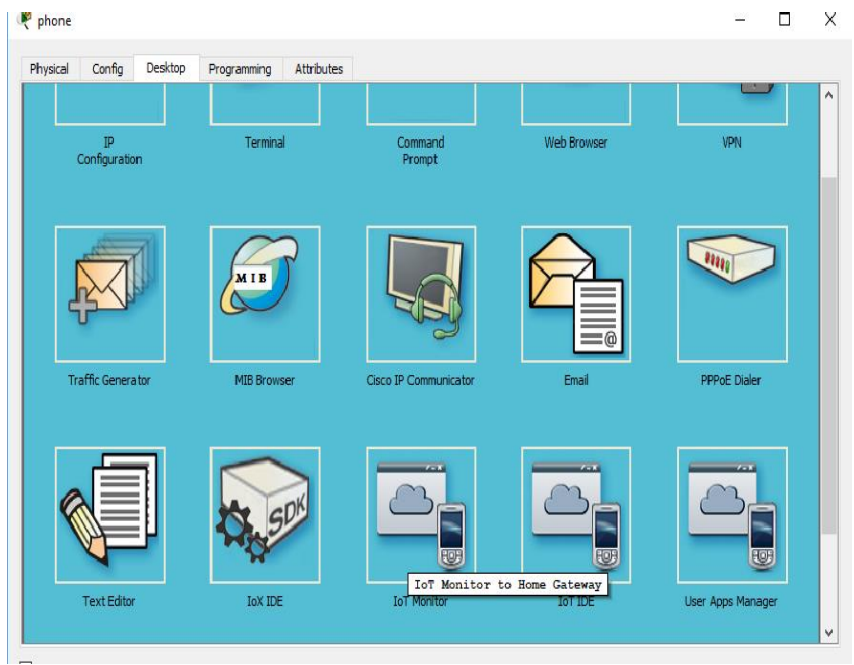

Fig.. 7: IOT monitor to home gateway.

When IOT monitor is selected, the rules can be added, click conditions then click add to add new activities such as lock door condition as shown in Fig 8 and Fig 9 shows the rules written for controlling door.

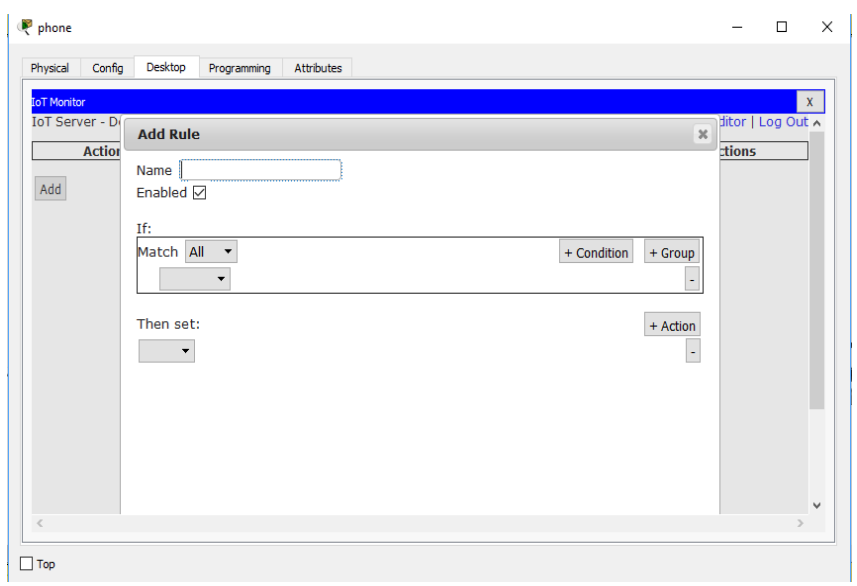

Fig. 8: Settings for adding rules.

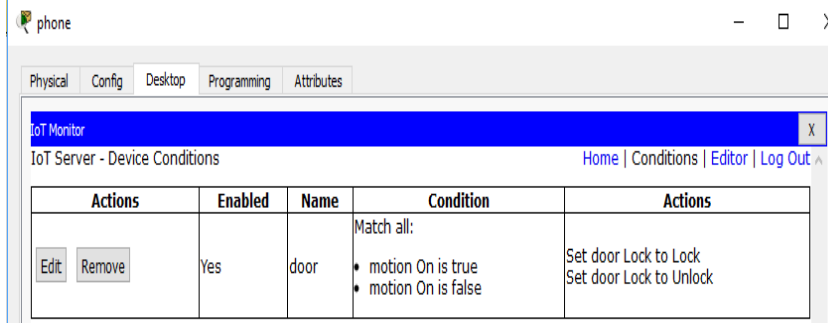

\section{Add}

จTop

Fig. 9: Door rules.

Different conditions and rules are programmed based on the smart object connected to home gateway. These steps have to be repeated for all objects.

\section{RESULTS}

Once you finish all the main procedure of designing a smart home environment that is - Add home gateway to the workspace, Add IoT devices to the home gateway in workspace and finally add the end user device such as smartphone, tab, laptop, etc. When the smartphone in the workspace is clicked, IoT server can be selected to verify the connections that have been established. Fig 10 shows the registered devices in the smart home implemented.

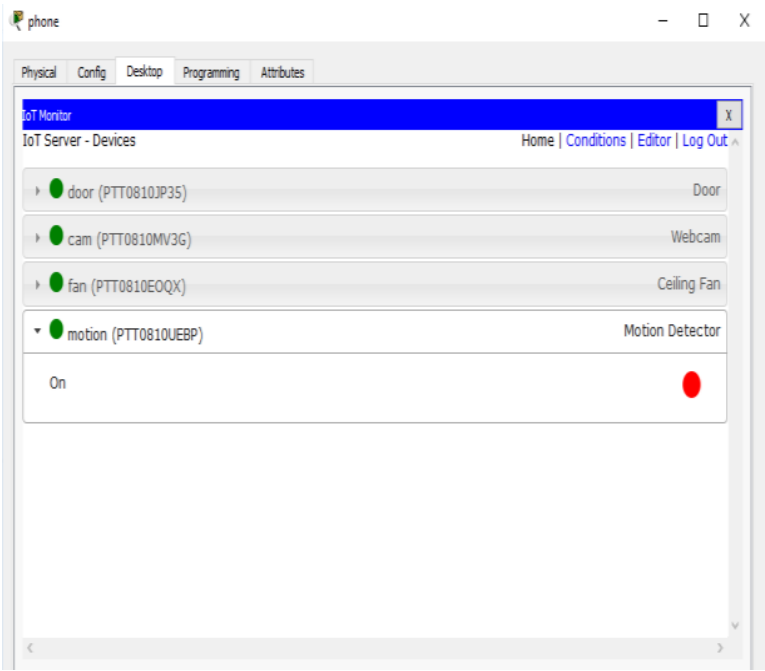

Fig. 10: Smart devices registered in network

\section{CONCLUSION AND FUTURE WORK}

The aim of this research was to simulate smart home. The technology innovation and the hike in smartphone usage was the motivation behind this work. The security measures are very critical and IoT is providing a new and excellent concept on make our surroundings smarter. 
In this research cisco packet tracer was utilized to design and simulate the smart home. Cisco public tutorials and previous researches were very beneficial in the implementation process. The result showed that the devices can be controlled and monitored using end user device. Cisco packet tracer gives various facilities which makes simulation easy.The results proves that there an opportunity to apply this model in real life. IoT concept can be applied in various domains.

\section{REFERENCES}

[1] Corporate Social Responsibility. (2018). Retrieved from https://www.cisco.com/c/en/us/about/csr.html

[2] packettracernetwork. (2018, January). (What's new in Cisco Packet Tracer 7.0) Retrieved from packettracernetwork: http://www.packettracernetwork.com/features/packettracer-7new-features.html

[3] A, E., \& K. A, H. (n.d.). Design and Implementation of a WiFi Based Home Automation System,. pages 2177-2180.

[4] Abdi, A. (2018). Designing Smart Campus Using Internet of Things. International Journal of Computer Science Trends and Technology (IJCST), V 6 I 3 PP 109-116.

[5] Aggarwal, R., \& Lal Das, M. (2012). RFID Security in the Context of Internet of Things. page 51-56. Kerala: First International Conference on Security of Internet of Things.

[6] al, M. c. (n.d.). Designing Smart Campus Using Internet of Things. 6(3).

[7] Alexandria, A. (2014). Enabling communication between wireless sensor networks and the internet of things-ACOAP communication stacks. International journal of Science and Engineering, 6-7 Vol.5.

[8] AlFarsi, G., \& ALSinani, M. (2017). Developing a Mobile Notification System for AlBuraimi University College Students. International Journal of Information Technology and Language Studies(IJITLS), 1(1).

[9] ALFarsi, G., Jabbar, J., \& ALSinani, M. (2018). Implementing a Mobile Application News Tool for Disseminating Messages and Events of AlBuraimi University College. International Journal of Interactive Mobile Technologies (iJIM), 12(7).

[10] ALFARSI, G., M, K. A., \& ALSINANI, M. (2017). A RULEBASED SYSTEM FOR ADVISING UNDERGRADUATE STUDENTS. Journal of Theoretical and Applied Information Technology, 95(11).

[11] A 1-Emran, M., Malik, S. I., \& Al-Kabi, M. N. (2020). A Survey of Internet of Things (IoT) in Education: Opportunities and Challenges. In Toward Social Internet of Things (SIoT): Enabling Technologies, Architectures and Applications (pp. 197-209). Springer, Cham

[12] Farahat, I. S., Tolba, A. S., Elhoseny, M., \& Eladrosy, W. (2019). Data security and challenges in smart cities. In Security in Smart Cities: Models, Applications, and Challenges (pp. 117-142). Springer, Cham

[13] Chasaki, D., \& Mansour, C. (2015). Security challenges in the internet of things. International Journal of Space-Based and Situated Computing, 5(3), 141-149.

[14] Mumtaz, S., Alsohaily, A., Pang, Z., Rayes, A., Tsang, K. F., \& Rodriguez, J. (2017). Massive Internet of Things for industrial applications: Addressing wireless IIoT connectivity challenges and ecosystem fragmentation. IEEE Industrial Electronics Magazine, 11(1), 28-33.

[15] Banafa, A. (2016). IoT standardization and implementation challenges. IEEE Internet of Things Newsletter.

[16] Priyan, M. K., \& Devi, G. U. (2019). A survey on internet of vehicles: applications, technologies, challenges and opportunities. International Journal of Advanced Intelligence Paradigms, 12(1-2), 98-119.

[17] Darwish, A., Hassanien, A. E., Elhoseny, M., Sangaiah, A. K., \& Muhammad, K. (2019). The impact of the hybrid platform of internet of things and cloud computing on healthcare systems: Opportunities, challenges, and open problems. Journal of Ambient Intelligence and Humanized Computing, 10(10), 4151-4166.

[18] Ghasempour, A. (2019). Internet of Things in Smart Grid: Architecture, Applications, Services, Key Technologies, and Challenges. Inventions, 4(1), 22. 